The ASTROPHYSICAL Journal, 285:L49-L52, 1984 October 15

1984. The American Astronomical Society. All rights reserved. Printed in U.S.A.

\title{
SPECTROSCOPY OF EXTREMELY DISTANT RADIO GALAXIES
}

\author{
Hyron SPINRAD $^{1}$ AND S. DJORgOvSKI ${ }^{2}$ \\ Department of Astronomy, University of California, Berkeley \\ Received 1984 June 7; accepted 1984 July 12
}

\begin{abstract}
In this Letter we report the firm identification of two faint radio galaxies, 3C 13 and $3 \mathrm{C} 256$, and a spectroscopic survey of 13 faint $3 \mathrm{CR}$ galaxies. The emission-line redshifts now extend up to $z=1.82$ (3C 256), and we here present a list and a preliminary spectrum summary of the character of 11 new radio galaxies with $z>0.77$. About half of these luminous galaxies show low ionization emission-line spectra. Their strong [O II] lines can have luminosities up to $9 \times 10^{43} \mathrm{ergs} \mathrm{s}^{-1}$; the emitted UV spectra have a much "softer" ionizing radiation field than those of QSOs and Seyfert nuclei. The spectra are of the narrow-line variety, with FWHM $\leq 1000 \mathrm{~km} \mathrm{~s}^{-1}$. The strong [O $\left.\mathrm{II}\right]$ emission is often much extended and inclined, showing a considerable velocity amplitude. The most extreme cases are 3C 324 and 3C 267 (both with $z>1.0$ ), but the phenomenon is moderately frequent. These are the most distant (non-QSO) galaxies known, and we can now extend the cosmological and galaxy evolution tests into previously unprobed territory.
\end{abstract}

Subject headings: radio sources: galaxies - radio sources: spectra

\section{INTRODUCTION AND OBSERVATIONS}

Over the last 2 years we have been obtaining long slit spectra of the faintest radio galaxies considered reasonably well identified (cf. Laing, Riley, and Longair 1983). The spectrograms were obtained with the Kitt Peak 4 m Mayall reflector using the cryogenic camera and two low-resolution grisms as dispersing devices (De Veny 1983). The two-dimensional extractions and one-dimensional spectroscopic reductions have been carried out in Berkeley (Djorgovski and Spinrad 1983). A long slit ensures sufficient signal and interpolative capability on the sky regions surrounding each faint galaxy (the galaxy spectrum itself is usually extracted from about 5 pixels [columns on the CCD chip], corresponding to 4 ".2 width on the sky).

Starting with the prime identification papers of Kristian, Sandage, and Katem $(1974,1978)$, Smith and Spinrad (1980), Gunn et al. (1981), and recently culminating with the summary of Laing, Riley, and Longair (1983), the number of faint galaxies and probable galaxies identified with the powerful $3 \mathrm{CR}$ radio sample has grown. Smith and Spinrad listed about 44 sources identified with faint $(V \geq 20)$ galaxies with no modern spectroscopic information. We have now improved that situation considerably; with the cryogenic camera we often obtain emission-line redshifts for $V=22$ galaxies in only an hour exposure! This same sort of positive result typifies the parallel spectroscopic effort of Perryman et al. (1984) who obtained five redshifts of other faint 3CR galaxies. The faintest galaxies for which we obtained spectra have $V \approx 23-24$.

\footnotetext{
${ }^{1}$ Visiting Astronomer, Kitt Peak National Observatory, National Optical Astronomy Observatory, operated by the AURA, Inc., under contract with the NSF.

${ }^{2}$ Visiting Student, Kitt Peak National Observatory, National Optical Astronomy Observatory, operated by the AURA, Inc., under contract with the NSF.
}

\section{NEW IDENTIFICATION AND IMAGING OF 3C 13} AND 3C 256

In the identification paper on $3 \mathrm{C} 13$ (Smith, Burbidge, and Spinrad 1976, hereafter SBS) it was noted that the $5 \mathrm{GHz}$ radio centroid of the unequal double radio source was displaced from the then suggested optical galaxy by some 5". Spectroscopy of this 20th mag candidate by Spinrad, Stauffer, and Butcher (1981) seemed to indicate that 3C 13 was a giant E galaxy with an absorption-line spectrum at $z=1.05$. Unfortunately, the 15 noisy co-added spectra of the initial 3C 13 candidate, taken with the Lick IDS scanner, do not tell the story correctly. Better KPNO cryogenic camera spectra of this galaxy show it to be at $z=0.477$, with weak [O II] emission and the usual $\lambda 4000$ discontinuity. So recently we have concentrated on the fainter galaxy to the north of the original candidate. Astrometry by M. Lebofsky places the "new" optical candidate for 3C 13 galaxy at:

$$
\alpha_{1950}=00^{\mathrm{h}} 31^{\mathrm{m}} 33^{\mathrm{s}} .17 ; \quad \delta_{1950}=+39^{\circ} 07^{\prime} 41^{\prime \prime} .78 .
$$

These coordinates agree well with the radio center positions (Fig. 1 [P1. L3]).

Spectroscopy of this fainter $(V=22)$ galaxy strongly confirms it as the correct identification; our Cryocam spectra show unusual strong and high-ionization UV emission lines at $z=1.351$ (Fig. $3[$ top $]$ ).

3C 256 has a simpler, but also interesting case history. The original finding chart (Kristian and Sandage 1970) was incorrectly marked (see the footnote in Smith and Spinrad 1980), but the correct (approximate) optical coordinates are given for this faint $(V \approx 22)$ galaxy. Again we observed it spectroscopically in advance of the modern imaging data. Our new direct image, shown here as Figure 2 (Plate L4), was recently obtained with the Mayall telescope and a new Texas Instruments $\mathrm{CCD}$ at the prime focus. Note the very elongated 
PLATE L3

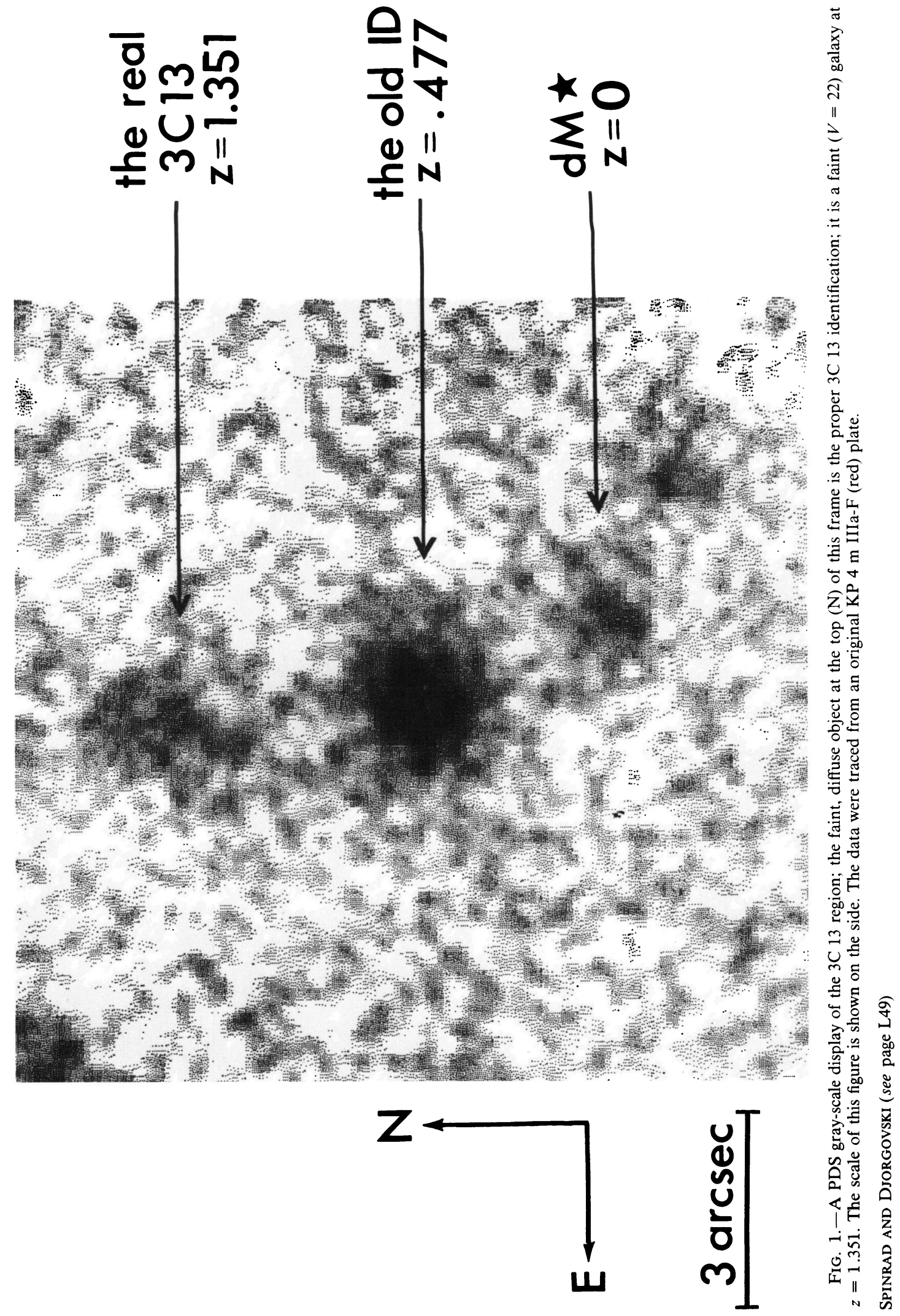




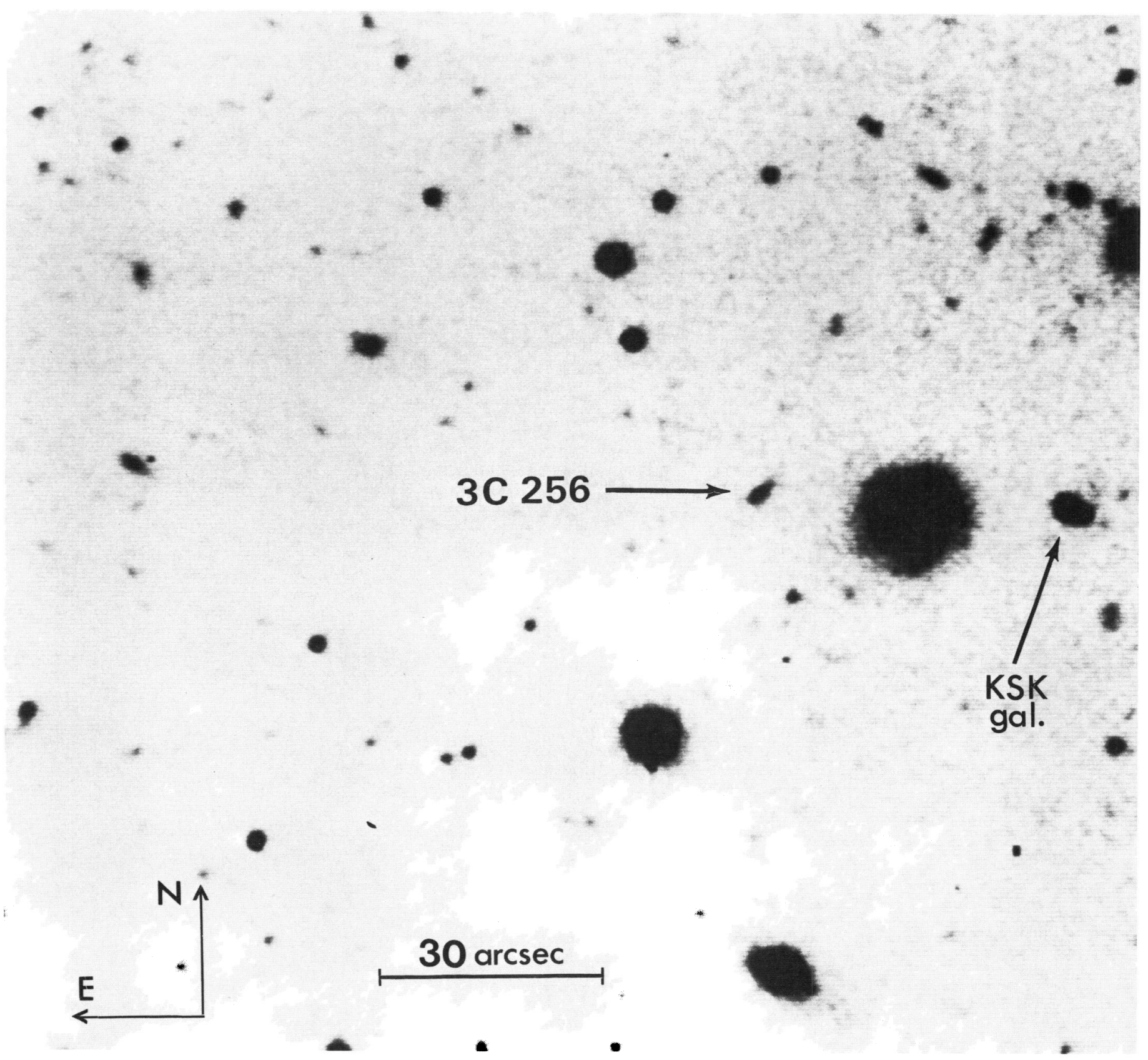

Fig. 2. A portion of an $R$-band CCD frame of $3 C 256$. The radio galaxy marked is the elongated (p.a. $=138^{\circ}$ ) image $18^{\prime \prime}$ from the large spiral we have called Wyndham's galaxy. Our new position for the center of $3 \mathrm{C} 256$ is $\alpha_{1950}=11^{\mathrm{h}}-18^{\mathrm{m}}-04^{\mathrm{s}} .25 \pm 00^{\mathrm{s}} 1, \delta_{1950}=+23^{\circ} 44^{\prime} 22^{\prime \prime} .6 \pm 0^{\prime \prime} .8$, and the coordinate zero point comes from the Wyndham galaxy position of Kristian and Sandage (1970). The double galaxy to the west of Wyndham's galaxy was noted by KSK (1978).

SpINRAD AND DJorgovski (see page L49) 

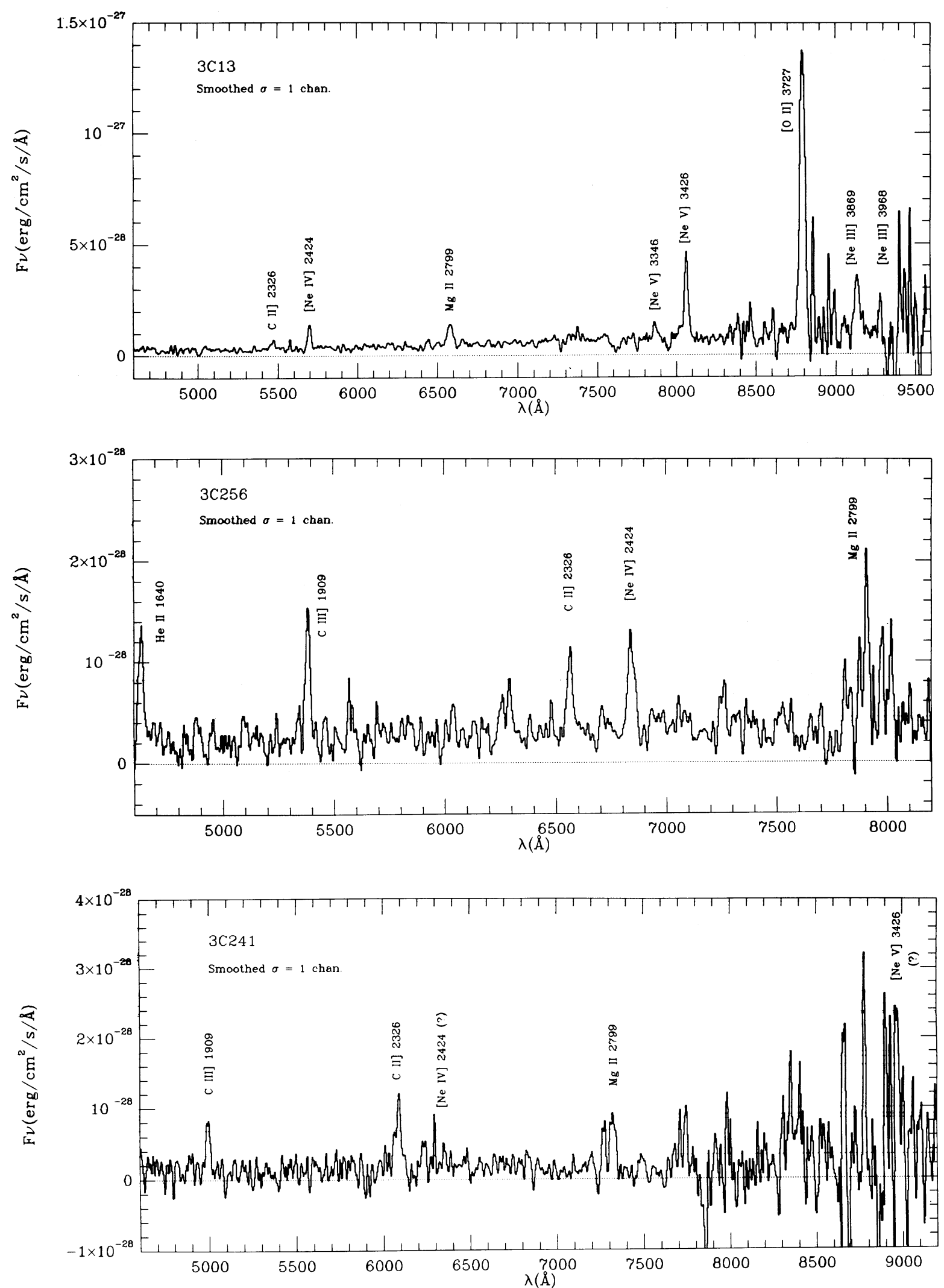

FIG. 3.-(top) The composite spectrum of $3 \mathrm{C} 13$, a high-ionization emission-line radio galaxy. Note the strength of [O II] $\lambda 3727$ in the infrared; this galaxy shows unusually strong [Ne IV] and [Ne v] lines. (middle) The spectrum of 3C 256 at $z_{e}=1.819$ shows UV emission lines of fairly great intensity, and intermediate ionization level. Note that the emission features are all quite narrow, even the permitted lines. This is the first detection of He II 1640 in the spectrum of a distant galaxy. The infrared end is noisy, because the sky is very bright at $\lambda>7700 \AA$. (bottom) The spectrum of the very faint galaxy associated with 3C 241 is of lower ionization, with C II] 2326 being the strongest emission line in the visible region. The noisy, doublet-like structure near $\mathrm{Mg}$ II is probably due to imprecise sky subtraction of the strong telluric $\mathrm{OH}$ band near $\lambda 7300 \AA$. 
appearance of 3C 256; it certainly does not resemble nearby powerful giant $\mathrm{E}$ radio sources! 3C 256 is important to study, as it now carries the largest measured redshift for a galaxy, $z=1.819$ (see below).

\section{REDSHIFTS AND EMISSION-LINE STRENGTHS FOR THE 3 CR GALAXIES}

Our spectroscopic task has been greatly simplified by the propensity of radio galaxies to show emission lines; this has been known for a long time with regard to the optical and near-ultraviolet regions $(\lambda>3400 \AA)$. But our knowledge of the emitted UV spectral region, $\lambda<3000 \AA$, in galaxies, as opposed to QSOs, is far more limited. Fortunately most luminous radio galaxies do have moderately strong low-ionization and "mixed-ionization" emission-line spectra. We report on 13 of those here; a few other objects with well-detected continua still stubbornly refuse to show any emission features

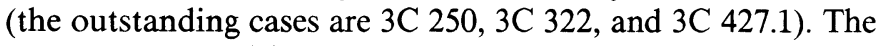
emission-line redshifts presented are deemed to be secure; they are always based upon at least two or (usually) three or four emission lines.

Table 1 lists the measured redshifts for 3C 324 (Spinrad and Djorgovski 1984), 3C 280 (Spinrad 1982), and 11 new radio galaxies so far reduced; the velocities are as observed; they have not been corrected for galactic rotation or reduced to a proper Local Group centroid velocity. The typical internal uncertainties in $z$ are about \pm 0.001 , but small systematic errors in the wavelength solutions with the Cryocam could lead to slightly larger external errors.

The spectra of 3C 256 and 3C 241, both at very large redshift, are notable. The spectrum of 3C 256 is shown as Figure 3 (middle). It shows fairly strong and narrow emission lines of He II 1640, C III] 1909, C II] 2326, [Ne v] 2424, and $\mathrm{Mg}$ II 2799. The FWHM for these lines is small, about 1000 $\mathrm{km} \mathrm{s}^{-1}$. 3C 256 is clearly a narrow-line radio galaxy. Other radio galaxies in our sample have FWHM ranging from 500 to $1200 \mathrm{~km} \mathrm{~s}^{-1}$, after an approximate correction for the convolution of the instrumental resolution.

3C 241's spectrum, shown in Figure 3 (bottom), is mostly of lower ionization; it shows $\mathrm{C}$ III] and $\mathrm{C}$ II] on the visible grism exposure. While weak [ $\mathrm{Ne} \mathrm{V}]$ appears at long wavelengths, 3C 241 appears to be an example of the common, relatively low-ionization emission spectrum often encountered in distant luminous radio galaxies (Spinrad 1982). Their spectra are far "softer" than almost all QSOs and Seyfert nuclei (cf. Davidson and Netzer 1979; Véron-Cetty, Véron, and Tarenghi 1983; Clavel and Joly 1984). The QSOs and Seyfert nuclei typically show C III] $\gg \mathrm{C} \mathrm{II}$ and rarely have measured [Ne IV] 2424; our galaxies often have $\mathrm{C}$ II] 2326 and [Ne IV] 2424 as two of the fairly intense ultraviolet emission lines. Several of our spectra show partial resolution of the $\mathrm{Mg}$ II doublet ( $\lambda \lambda 2795,2802 \AA)$ - thus reaffirming their narrow-line nature. 3 C 13's spectrum (Fig. 3 [top]) has a higher mean ionization than $3 \mathrm{C} 241$.
TABLE 1

LARGE REDShIFTS FOR DisTant 3CR GalaXies

\begin{tabular}{|c|c|c|}
\hline 3C No. & $z_{e}$ & Emission Lines Used \\
\hline 256. & 1.819 & Mg II, [Ne IV], C II], C III], He II \\
\hline $241 .$. & 1.617 & {$[\mathrm{Ne} \mathrm{V}], \mathrm{Mg}$ II, C II], C III] } \\
\hline $238 \ldots$ & 1.405 & [O II], Mg II \\
\hline $13 .$. & 1.351 & [Ne III], [O II], ]Ne v], Mg II, [Ne IV], C II] \\
\hline 469.1 & 1.336 & [O II], [Ne v], Mg II (weak) \\
\hline $266 .$. & 1.272 & [O II], [Ne v], $\mathrm{Mg}$ II, C II] \\
\hline $324 .$. & 1.2063 & [Ne III], [O II], Mg II, [Ne IV] (weak), C II] \\
\hline $267 .$. & 1.144 & {$[\mathrm{O}$ II $],[\mathrm{Ne} \mathrm{V}]$} \\
\hline $252 .$. & 1.105 & {$[\mathrm{Ne}$ v], Mg II, [Ne IV], C II } \\
\hline $280 .$. & 0.996 & {$[\mathrm{Ne} \mathrm{III}],[\mathrm{O} \mathrm{II}],[\mathrm{Ne} \mathrm{v}]$} \\
\hline $289 .$. & 0.9674 & {$[\mathrm{O} \mathrm{II}],[\mathrm{Ne} \mathrm{v}], \mathrm{Mg}$ II } \\
\hline 217. & 0.8975 & [Ne III], [O II], [Ne v], Mg II \\
\hline $340 \ldots$ & 0.7754 & {$[\mathrm{Ne} \mathrm{III}],[\mathrm{O} \mathrm{II}],[\mathrm{Ne} \mathrm{V}]$} \\
\hline
\end{tabular}

The [O II] $\lambda_{0} 3737$ lines in some of our galaxies $(z<1.5)$ are very strong; the observed line flux for $3 \mathrm{C} 266$, for example, is $4.1 \times 10^{-15} \mathrm{ergs} \mathrm{cm}^{-2} \mathrm{~s}^{-1}$; converting to an [O II] luminosity (as in Spinrad 1982) yields $L_{[\mathrm{O}}{ }_{\mathrm{II}}=7.5 \times 10^{43} \mathrm{ergs} \mathrm{s}^{-1}$ in this one emission line! Four of the galaxies in our reduced sample have $L_{[\mathrm{O}}{ }_{\mathrm{II}]}>5 \times 10^{43} \mathrm{ergs} \mathrm{s}^{-1}\left(\right.$ or $\left.>1 \times 10^{10} L_{\odot}\right)$ in $\lambda_{0} 3727$ emission. However, not all the ionized gas is confined to the galaxy nucleus. To our surprise, several of those distant radio galaxies, observed initially at arbitrary telescopic position angles, showed an extended [O II] feature, typically $\sim 4^{\prime \prime}$ in length, with a velocity gradient or at least obvious velocity structure, only partly resolved with our $1^{\prime \prime}-2^{\prime \prime}$ seeing smear. The data on the extended $\lambda_{0} 3727$ line for 3C 324 have been published (Spinrad and Djorgovski 1984). We defer further interpretation until our direct imaging program catches up closer to our spectroscopy.

It is our intention to discuss the emission-line intensities and their physical interpretation, and the hints about early galaxy evolution from the shapes of these luminous radio galaxies, in full papers to follow. The present set of galaxies extends the available redshift range for cosmological and evolution tests into previously uncharted territory to look-back times of the order of $60 \%-70 \%$ of the Hubble time. In the meantime we are continuing observations of even fainter radio galaxy candidates to the present equipmental limit. Who knows what will be found?

We are very thankful to the staff of the KPNO for the valuable help in our observations, and in particular to $\mathrm{J}$. De Veny, W. Ditsler, J. Barnes, S. Hammond, and G. Will. We also thank our colleagues M. Lebofsky, R. Laing, J. Kristian, and especially S. Lilly, for communication of positions and encouragement. Some of the data reductions were performed by J. Marr, and some of the software written by P. Monger. This work was partly supported by NSF grant AST81-16125 to H. Spinrad and a University of California Graduate fellowship to S. Djorgovski.

\section{REFERENCES}

Clavel, J., and Joly, M. 1984, Astr. Ap., 131, 87.

Davidson, K., and Netzer, H. 1979, Rev. Mod. Phys., 51, 715

De Veny, J. 1983, An Observer's Manual for the Cryogenic Camera, Kitt Peak National Observatory.
Djorgovski, S., and Spinrad, H. 1983, in Joint Topical Meeting on Information Processing in Astronomy and Optics, Minneapolis-St. Paul (OSA/AAS Publication), p. ThB2-1. 
Gunn, J. E., Hoessel, J. G., Westphal, J. A., Perryman, M. A. C., and Longair, M. S. 1981, M.N.R.A.S., 194, 111.

Kristian, J., and Sandage, A. 1970, Ap. J., 162, 391.

Kristian, J., Sandage, A., and Katem, B. 1974, Ap.J., 191, 43. 1978, Ap. J., 219, 803 (KSK).

Laing, R. A., Riley, J. M., and Longair, M. S. 1983, M.N.R.A.S., 204, 151.

Perryman, M. A. C., Lilly, S. J., Longair, M. S., and Downes, A. J. B. $1984, M . N . R . A . S .$, in press.

S. DJorgovski and Hyron SpinRad: Department of Astronomy, University of California, Berkeley, CA 94720
Smith, H. E., Burbidge, E. M., and Spinrad, H. 1976, Ap. J., 210, 627 (SBS).

Smith, H. E., and Spinrad, H. 1980, Pub. A.S.P., 92, 553 (SS).

Spinrad, H. 1982, Pub. A.S.P., 94, 397.

Spinrad, H., Stauffer, J., and Butcher, H. 1981, Ap. J., 244, 382.

Spinrad, H., and Djorgovski, S. 1984, Ap. J. (Letters), 280, L9.

Véron-Cetty, M. P., Véron, P., and Tarenghi, M. 1983, Astr. Ap., 119, 69. 